\title{
Opioid Use Is Reduced in Patients Treated with NSAIDs After Arthroscopic Bankart Repair: A Randomized Controlled Study
}

\author{
Kamali A. Thompson, B.S., M.B.A., David Klein, D.O., Michael J. Alaia, M.D., \\ Eric J. Strauss, M.D., Laith M. Jazrawi, M.D., and Kirk A. Campbell, M.D.
}

\begin{abstract}
Purpose: To evaluate the efficacy of oral nonsteroidal anti-inflammatory drugs (NSAIDs) as the primary postoperative pain medication compared with standard oral opioids after arthroscopic shoulder capsulolabral (Bankart) repair for recurrent anterior shoulder instability. Methods: This was a single-center, prospective, randomized controlled study. Patients aged 18 to 65 years indicated for arthroscopic shoulder capsulolabral repair for recurrent anterior shoulder instability were included. Postoperatively, patients were prescribed 1 of 2 analgesic regimens: (1) 30 ibuprofen (600 mg every 6 to 8 hours as needed) and 10 tablets of oxycodone/acetaminophen (5/325 mg every 6 hours as needed for breakthrough pain) or (2) 30 tablets oxycodone/acetaminophen (5/325 mg every 6 hours as needed). Subjects completed questionnaires at 24, 48, and 96 hours and 1 week after surgery, which included questions about analgesic medication usage, visual analog scale (VAS) pain score, incidence of adverse events, and patient satisfaction. Results: Between December 2017 and May 2019, 80 patients (mean age 31.3 \pm 10.4 years) were enrolled. Overall there were no significant differences in VAS pain score or patient satisfaction between the 2 groups. Patients in the opioid-only group had a significantly higher mean opioid consumption on postoperative days (PODs) 2 to $4(5.5$ versus $2.9, P=.05)$ and after 1 week (11.7 versus 7.9 tablets, $P=.05)$ compared with patients in the NSAID group. Of patients in the NSAID group, $17.5 \%$ experienced adverse effects, compared with 35\% in the opioid-only group. Conclusions: Use of oral NSAIDs with limited breakthrough opioids results in significantly less opioid use after arthroscopic Bankart repair in the first postoperative week compared with opioids only. Both groups used limited amounts of opioids to control postoperative pain. There were no differences in pain levels at any time point postoperatively or satisfaction between patient groups. Level of Evidence: II, nonblinded randomized control trial.
\end{abstract}

S houlder instability is a common indication for arthroscopic surgery in active patients. Surgical stabilization is becoming more common in recent years, with the incidence of arthroscopic stabilization codes being reported increasing by $8 \%$ per year, and open stabilization increasing by $15 \%$ per year. ${ }^{1}$ As an outpatient procedure, patients must

From the Division of Sports Medicine, Department of Orthopedic Surgery, NYU Langone Orthopedic Hospital, NYU Langone Health, New York, New York.

The authors report that they have no conflicts of interest in the authorship and publication of this article. Full ICMJE author disclosure forms are available for this article online, as supplementary material.

Received January 2, 2020; accepted August 7, 2020.

Address correspondence to Kamali Thompson, B.S., M.B.A., 333 E 38th St, New York, NY 10016, U.S.A. E-mail: kamali.thompson@nymc.org

(C) 2020 THE AUTHORS. Published by Elsevier Inc. on behalf of the Arthroscopy Association of North America. This is an open access article under the CC BY-NC-ND license (http://creativecommons.org/licenses/by-nc-nd/4.0/). 2666-061X/2011

https://doi.org/10.1016/j.asmr.2020.08.003 be discharged home with adequate analgesia for pain control in the early postoperative period. Multimodal pain control has become a common approach, with a combination of nerve blockade, cryotherapy, oral analgesics such as acetaminophen and nonsteroidal anti-inflammatory drugs (NSAIDs), and oral opioids.

The current opioid epidemic in the United States is closely related to physician prescribing habits, with orthopedic surgeons noted to be the third-highest opioid prescribers nationally. ${ }^{2}$ As surgeons have come to recognize the gravity of the opioid epidemic, there has been a push to avoid using narcotics whenever possible, since opioid abuse is closely tied to prescription opioids. $^{3}$ Patients who abuse oral opioids are 40 times more likely to abuse harder drugs such as heroin. ${ }^{4,5}$ Even worse, it has been estimated that the majority of prescribed opioids are unused, not stored properly, and available for abuse by patients and their family members. ${ }^{6}$ 


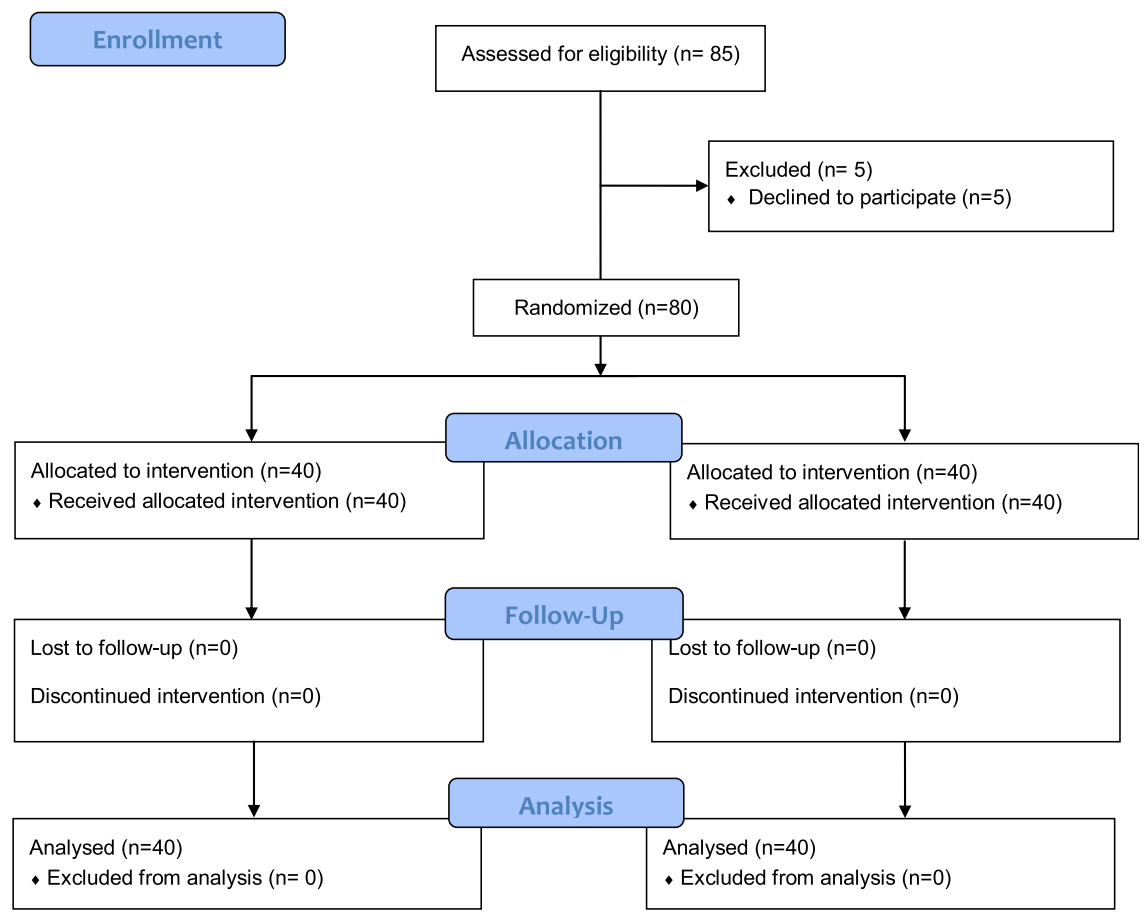

Fig 1. Consort diagram (Consort Transparent Reporting of Trials).
The American Academy of Orthopedic Surgeons has called for evidence-based use of opioids, and strides have been made in the total joint arthroplasty and hip arthroscopy literature. ${ }^{7-11}$ Arthroscopic shoulder capsulolabral (Bankart) repair for anterior shoulder instability is typically performed as an outpatient procedure, with short surgical times and minimal soft tissue dissection and bony management. The use of nonopioid pain control in the ambulatory setting after arthroscopic shoulder capsulolabral (Bankart) repair has not been thoroughly explored.

The purpose of the current study was to evaluate the efficacy of oral NSAIDs as the primary postoperative pain medication compared with standard oral opioids after arthroscopic shoulder capsulolabral (Bankart) repair for recurrent anterior shoulder instability. We hypothesized that prescribing an NSAID as the primary pain medication, along with an opioid for breakthrough pain, could reduce overall opioid consumption and provide similar pain control and patient satisfaction postoperatively versus an opioid alone.

\section{Methods}

\section{Study Design}

This was a single-center, prospective, randomized controlled trial evaluating postoperative pain levels and opioid consumption between patients prescribed either predominantly NSAIDs or opioids only for analgesia after arthroscopic Bankart repair. All primary diagnoses were recurrent anterior shoulder instability with a confirmed Bankart tear on magnetic resonance imaging. The primary procedure of all enrolled patients was arthroscopic Bankart repair. Procedures were performed by 5 sports medicine fellowship-trained surgeons.

After institutional review board approval, patients who met inclusion criteria between December 2017 and May 2019 were enrolled (Fig 1). Inclusion criteria included subjects ages 18 to 65 years who were indicated for an arthroscopic shoulder capsulolabral repair for recurrent anterior instability. Exclusion criteria included subjects with contraindications to ibuprofen or oxycodone/acetaminophen (which included medical history of hypersensitivity reaction, liver disease, severe renal disease, gastrointestinal bleeding disorder, coagulopathy, or drug abuse) and patients taking opioid medication before surgery. Additionally, any patients requiring other concurrent open procedures were excluded. Patients requiring secondary arthroscopic procedures (remplissage, subacromial decompression, rotator cuff repair, distal clavicle resection, etc.) were included in this study.

Patients were randomized by medical record number (MRN) to receive 1 of 2 oral medication regimens for postoperative analgesia. Odd MRNs received 30 tablets of ibuprofen (600 mg every 6 to 8 hours as needed) and 10 tablets of oxycodone/acetaminophen $(5 / 325 \mathrm{mg})$ to be used every 6 hours as needed for breakthrough pain relief. Even MRNs received 30 tablets of oxycodone/ acetaminophen (5/325 mg every 6 hours as needed). Patients in the oxycodone/acetaminophen group were 
Table 1. Patient characteristics

\begin{tabular}{|c|c|c|c|}
\hline Characteristic & NSAID Group $(\mathrm{n}=40)$ & Opioid-Only Group $(\mathrm{n}=40)$ & $P$ Value \\
\hline$\overline{\text { Age }(y)}$ & $29.9 \pm 10.1$ & $32.6 \pm 10.8$ & .25 \\
\hline Body mass index $\left(\mathrm{kg} / \mathrm{m}^{2}\right)$ & $24.8 \pm 5.9$ & $25.4 \pm 3.9$ & .62 \\
\hline \multicolumn{4}{|l|}{ Sex } \\
\hline Male & $27(67.5)$ & $25(62.5)$ & .64 \\
\hline \multicolumn{4}{|l|}{ Smoking } \\
\hline Nonsmoker & $32(80)$ & $32(80)$ & 1.0 \\
\hline Anchors & $3.5 \pm 1.1$ & $3.3 \pm 1.1$ & .53 \\
\hline \multicolumn{4}{|l|}{ Concomitant procedure } \\
\hline Total patients & 15 & 21 & .18 \\
\hline Labral debridement & 10 & 6 & .26 \\
\hline Rotator cuff debridement & 3 & 11 & .01 \\
\hline Rotator cuff repair & 1 & 1 & 1.0 \\
\hline Bursectomy & 5 & 8 & .36 \\
\hline Synovectomy & 4 & 6 & .50 \\
\hline Remplissage & 1 & 1 & 1.0 \\
\hline Loose body removal & 2 & 0 & .15 \\
\hline
\end{tabular}

not restricted to using only their prescribed study medication; if they chose to use an over-the-counter (OTC) analgesic, the type and quantity of the medication was recorded at each time point throughout the study. However, patients were not encouraged to seek outside OTC medications.

\section{Demographic Data and Clinical Outcomes}

Demographic and surgical data were recorded prospectively. Demographic data included age, sex, height, weight, and body mass index (BMI). Surgical data included procedure, number of anchors used, concomitant procedures, and type of anesthesia given (Table 1). Patients were instructed to contact their surgeon if their pain was not controlled by either medication or if they needed a refill of medication.

All procedures were performed under regional anesthesia in the form of an interscalene nerve block with general anesthesia from the anesthesiologist. Intraoperative narcotic dosages were the same for both

Table 2. Postoperative pain and medication consumption

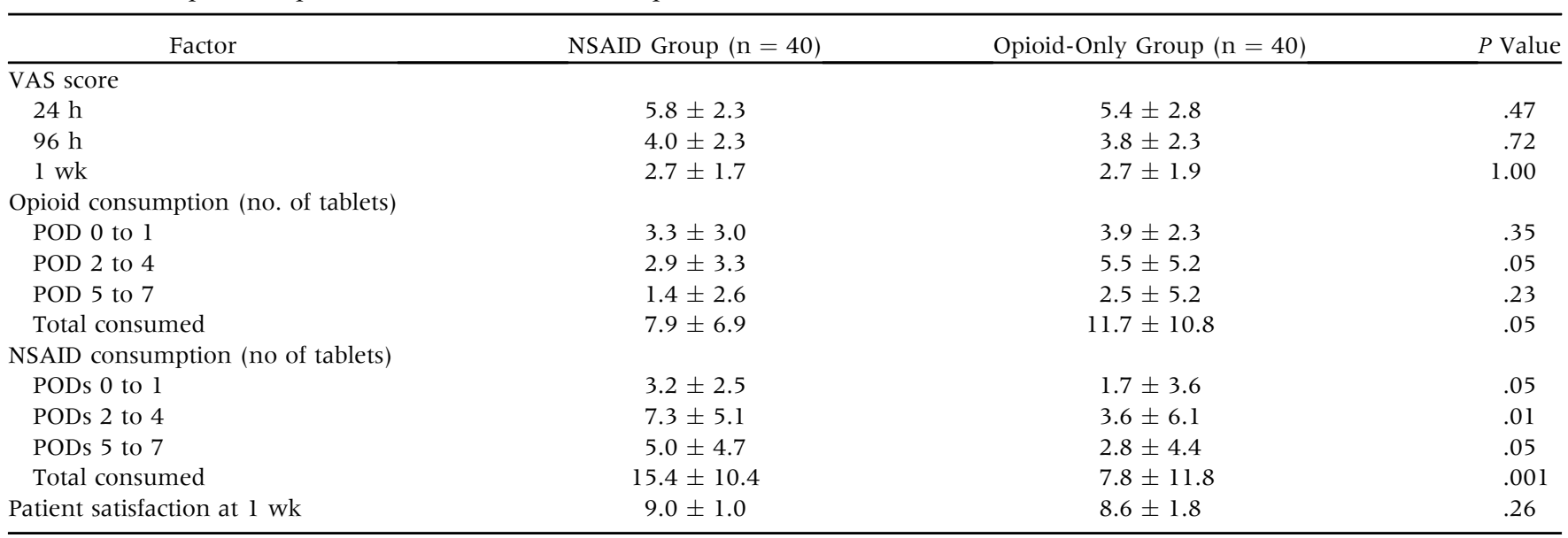

Data are mean \pm standard deviation.

NSAID, nonsteroidal anti-inflammatory drug; POD, postoperative drug. 
Table 3. Adverse effects experienced POD 0-7

\begin{tabular}{lccc}
\hline \multicolumn{1}{c}{ Effect } & PODs 0 to 1 & PODs 2 to 4 & POD 5 to 7 \\
\hline NSAID group & 2 & 0 & 0 \\
$\quad$ Nausea & 2 & 0 & 0 \\
Nausea and vomiting & 0 & 3 & 2 \\
$\quad$ Constipation & 3 & 2 & 0 \\
Opioid group & 2 & 0 & 0 \\
$\quad$ Nausea & 2 & 2 & 0 \\
Nausea and vomiting & 4 & 3 & 3 \\
Vomiting & & & \\
Constipation & &
\end{tabular}

groups, with no significant differences. No patients were given preoperative or intraoperative NSAIDs or acetaminophen. Patients were given reusable gel ice packs for postoperative care at home. All patients used a sling for $\sim 4$ weeks. Physical therapy was started after the first postoperative visit, which typically occurred 1 week after surgery.

After discharge, patients were contacted via telephone by a trained research associate uninvolved in their care to record medication usage (day of surgery [DOS], postoperative days [PODs] 0 to 1, PODs 2 to 4, PODs 5 to 7 , and 1 week total) as well as visual analog scale (VAS) pain scores (scale from 0 to 10 , with 0 indicating no pain and a score of 10 indicating severe, debilitating pain), incidence of adverse effects (nausea, vomiting, constipation, pruritus, numbness, weakness, and falls), and patient satisfaction (scale from 1 to 10 , 10 being completely satisfied and 1 being least satisfied) 1 week after surgery (Appendix). Patients brought their medication bottles to their l-week postoperative follow-up to confirm the amount of medication remaining.

\section{Statistical Analysis}

A priori power analysis was performed to detect a difference with an effect size of 0.40 using an $\alpha$ of 0.05 and a $\beta$ of 0.80 . The analysis determined that a minimum sample size of 64 patients ( 32 in each group) was needed. Statistical analysis was performed using SPSS statistical software version 24 (IBM, Armonk, NY). Differences between the 2 analgesic regimens were analyzed using independent-samples $t$ tests for continuous variables and chi-squared tests for categorical variables. Linear regression analyses were done to assess for correlations among variables. Findings were considered significant if $P \leq .05$.

\section{Results}

A total of 80 consecutive patients were enrolled, 40 of whom were prescribed regimen 1 (NSAID group) and 40 prescribed regimen 2 (opioid-only group) (Figure 1).
The mean age was $29.9 \pm 10.1$ years for the NSAID group and $32.6 \pm 10.8$ years for the opioid-only group, and mean BMI was $24.8 \pm 5.9$ for the NSAID group and $25.4 \pm 3.9$ for the opioid-only group (Table 1).

There was no difference between groups in age, BMI, the type of anesthesia used, the number of anchors inserted, or duration of surgery. There was no correlation between age, sex, smoking, or BMI and VAS score or opioid consumption in either group. No patient asked for a refill of medication, and all patient-reported information correlated with the number of pills in the bottle at the follow-up visit.

There was no statistically significant difference in the number of patients who underwent concomitant procedures between cohorts (Table 1). Although the opioid-only group had statistically significantly more rotator cuff debridements than the NSAID group (11 versus $3, P=.01$ ), there was no difference in VAS between patients with and without concomitant procedures in either cohort.

The NSAID group reported slightly higher VAS scores throughout the week, although none of the differences were statistically significant (e.g., 24 hours postoperatively, VAS $5.8 \pm 2.3$ versus $5.4 \pm 2.8, P=.47$ ) (Table 2 ). The opioid-only group consistently showed a higher usage of oxycodone/acetaminophen throughout the week, especially PODs 2 to 4 (2.9 versus 5.5 tablets, $P=.05)$, a difference that reached statistical significance (Table 2). The opioid-only group also showed a higher amount of opioid consumption after 1 week (11.7 versus 7.9 tablets, $P=.05)$. Patients reported similar scores of patient satisfaction after 1 week $(P=.26)$.

Twenty patients $(50 \%)$ in the opioid-only group chose to take an OTC NSAID in addition to the prescribed oxycodone/acetaminophen solely because they wanted to minimize or avoid the use of narcotics of their own accord. Fourteen patients reported using 200 mg ibuprofen, and 6 patients used $220 \mathrm{mg}$ naproxen.

The NSAID group reported 3 side effects,- - nausea, vomiting, and constipation-by 7 patients (17.5\%) (Table 3). On POD 1, 2 patients reported nausea alone, and 2 patients reported nausea and vomiting. Constipation was reported on POD 4 by 3 patients and on POD 7 by 2 patients. There were no additional side effects throughout the week. Fourteen (35\%) patients in the opioid-only group reported side effects. Three patients reported nausea alone on POD 1, 2 patients reported nausea and vomiting, and 2 patients reported vomiting alone. On POD 4, 2 patients reported nausea, and 2 patients reported vomiting. Regarding constipation, 4 patients experienced this side effect on POD 1 , and 3 patients on PODs 4 and 7 . No patients experienced any excessive bleeding throughout the study.

\section{Discussion}

The results of the current study support our hypothesis that prescribing an NSAID for primary pain control 
along with an opioid for breakthrough pain provides equivalent postoperative analgesia and patient satisfaction while reducing opioid consumption compared with prescribing opioids alone. Patients prescribed NSAIDs and opioids for breakthrough pain had no difference in the extent of their pain or satisfaction throughout the first postoperative week. Overall, the NSAID group experienced pain levels similar to those of the oxycodone/acetaminophen group but used significantly less opioid medication than was prescribed. Seven patients $(17.5 \%)$ in the opioid-only group did use not any oxycodone/acetaminophen. An additional 10 patients $(25 \%)$ used $\leq 4$ oxycodone/acetaminophen throughout the entire week. These results indicate that postoperative pain after surgery for labral repair can be effectively controlled with OTC analgesics and limited quantities of opioid analgesics.

Several other studies have also documented the efficacy of nonopioid oral medications after arthroscopy and other orthopaedic procedures. ${ }^{12-19}$ Gimbel et al. ${ }^{14}$ studied a variety of orthopaedic procedures and concluded that celecoxib may serve as a better option for postoperative pain control compared with hydrocodone/acetaminophen. A recent study by Pham et al. ${ }^{19}$ showed that use of oral NSAIDs (ibuprofen) and limited narcotics resulted in excellent pain relief and patient satisfaction after arthroscopic meniscectomy, which supports our current findings for limited narcotics after an arthroscopic procedure. Although our study used ibuprofen, other investigators have suggested a variety of viable nonopioid options such as acetaminophen, transdermal buprenorphine, and cannabinoids. ${ }^{20-22}$ Nonopioid medications are a major component of multimodal analgesia, which is gaining popularity in the orthopedic community because of its opioid-sparing effect. ${ }^{23-25}$ Additionally, recent investigations into various non-narcotic perioperative medications have shown the potential to further reduce a patient's overall need for pain medicine in the immediate postoperative period and improve outcomes. . $^{19,26,27}$

Notably, our results of the mean l-week total opioid consumption showed that both NSAID and opioidonly groups used only a fraction of their prescribed amount ( 7.9 of 10 and 11.7 of 30 , respectively). This suggests that opioids were overprescribed in our study, a phenomenon that has been shown to contribute to the opioid epidemic. Furthermore, 50\% of the opioid cohort reported that they chose to take an OTC NSAID in addition to oxycodone/acetaminophen to decrease or avoid opioid consumption. This is supported in the literature, that the vast majority of patients undergoing outpatient orthopedic surgery consume significantly fewer opioids than they are prescribed. ${ }^{11,28,29}$ It is believed that almost half of all prescription drug abusers taking pain relievers obtain the medication from a friend or family member with excess medication, instead of a health care professional. ${ }^{30}$ Our findings highlight the need for additional research exploring the role of patient education about opioid reliability and handling before common orthopaedic procedures.

We cannot forget that NSAIDs also have the potential for adverse effects. Although NSAIDs are an effective method of pain control, they are associated with gastrointestinal hemorrhage and ulceration, changes in platelet function, and renal complications. ${ }^{14,31}$ Therefore, patients should be screened for contraindications or history of adverse reactions before being prescribed NSAIDs for postoperative analgesia. There is also controversy over the use of NSAIDs after orthopedic procedures, with several studies indicating that NSAIDs inhibit osteogenesis and delay bone healing. ${ }^{12,13,32,33}$ We consider it good practice to recommend taking NSAIDs along with food and to maintain proper hydration to minimize the risk of gastrointestinal and renal complications.

A limitation to fieldwide change in prescribing habits is the willingness to be inconvenienced by a patients in pain or having to spend more time on educating patients about opioids. Further investigation is required to determine whether surgeons would prefer to refill prescriptions for patients with excessive postoperative pain or continue the current guidelines. As the opioid epidemic gains public attention ${ }^{34}$ and institutions standardize their postoperative pain management protocols, it is imperative that surgeons set expectations for postoperative pain and attempt to supplement opioid narcotics with safer analgesics, even if this means the inconvenience of refilling prescriptions for patients with analgesic needs that surpass the initial prescribed amount.

The strengths of this study include that it was a randomized controlled design, had close follow-up, and involved a pill count to confirm the amount of narcotic medication used during the study period. Additionally, we included all patients whose primary diagnosis was shoulder instability regardless of any concomitant procedures performed at the time of the labral repair, making the findings more generalizable given that in clinical practice, we do address concomitant pathology. That patient characteristics were similar between the groups is another strength.

\section{Limitations}

There were several limitations to our study. First, it was a single-center study in which neither the patients nor the surgeons were blinded to the medication prescribed. Furthermore, patients were followed for only the first week after surgery. We did not explore whether narcotic requirements changed after that time period. Last, we did not measure pain preoperatively 
and were unable to determine how pain changed after the procedure.

\section{Conclusion}

Prescription of oral NSAIDs with limited breakthrough opioids results in significantly less opioid use after arthroscopic Bankart repair in the first postoperative week compared with opioids alone. Both groups used limited amounts of opioids to control postoperative pain. There were no differences in pain levels at any time point postoperatively or patient satisfaction.

\section{References}

1. Riff AJ, Frank RM, Sumner S, et al. Trends in shoulder stabilization techniques used in the United States Based on a large private-payer database. Orthop J Sports Med 2017;5:2325967117745511.

2. Volkow ND, McLellan TA, Cotto JH, Karithanom M, Weiss SR. Characteristics of opioid prescriptions in 2009. JAMA 2011;305:1299-1301.

3. Unick GJ, Rosenblum D, Mars S, Ciccarone D. Intertwined epidemics: national demographic trends in hospitalizations for heroin- and opioid-related overdoses, 19932009. PLoS One 2013;8:e54496.

4. Jones CM, Logan J, Gladden RM, Bohm MK. Vital Signs: Demographic and substance use trends among heroin users-United States, 2002-2013. MMWR Morb Mortal Wkly Rep 2015;64:719-725.

5. Lucyk SN, Nelson LS. Toxicosurveillance in the US opioid epidemic. Int J Drug Policy 2017;46:168-171.

6. Morris BJ, Mir HR. The opioid epidemic: Impact on orthopaedic surgery. J Am Acad Orthop Surg 2015;23: 267-271.

7. American Academy of Orthopaedic Surgeons. Information Statement 1045: Opioid use, misuse, and abuse in orthopaedic practice. Rosemont, IL: American Academy of Orthopaedic Surgeons, 2015.

8. Rodgers J, Cunningham K, Fitzgerald K, Finnerty E. Opioid consumption following outpatient upper extremity surgery. J Hand Surg 2012;37:645-650.

9. Cancienne JM, Patel KJ, Browne JA, Werner BC. Narcotic use and total knee arthroplasty. J Arthroplast 2018;33: 113-118.

10. Cunningham D, Lewis B, Hutyra C, Nho S, Olson S, Mather R. Prospective, observational study of opioid use after hip arthroscopy for femoroacetabular impingement syndrome. Arthroscopy 2018;34:1488-1497.

11. Kim N, Matzon JL, Abboudi J, et al. A prospective evaluation of opioid utilization after upper-extremity surgical procedures: Identifying consumption patterns and determining prescribing guidelines. J Bone Joint Surg Am 2016;98:e89.

12. Bali C, Ergenoglu P, Ozmete O, et al. Comparison of the postoperative analgesic effects of naproxen sodium and naproxen sodium-codeine phosphate for arthroscopic meniscus surgery. Braz J Anesthesiol 2016;66:151-156.

13. Drez D Jr, Ritter M, Rosenberg TD. Pain relief after arthroscopy: Naproxen sodium compared with propoxyphene napsylate with acetaminophen. South Med J 1987;80:440-443.

14. Gimbel JS, Brugger A, Zhao W, Verburg KM, Geis GS. Efficacy and tolerability of celecoxib versus hydrocodone/acetaminophen in the treatment of pain after ambulatory orthopedic surgery in adults. Clin Ther 2001;23:228-241.

15. Gimbel JS, Walker D, Ma T, Ahdieh H. Efficacy and safety of oxymorphone immediate release for the treatment of mild to moderate pain after ambulatory orthopedic surgery: Results of a randomized, double-blind, placebocontrolled trial. Arch Phys Med Rehabil 2005;86:2284-2289.

16. Ahn S, Byun SH, Park K, Ha JL, Kwon B, Kim JC. Analgesic efficacy of preemptive pregabalin administration in arthroscopic shoulder surgery: A randomized controlled trial. Can J Anaesth 2016;63:283-289.

17. Adam F, Menigaux C, Sessler DI, Chauvin M. A single preoperative dose of gabapentin (800 milligrams) does not augment postoperative analgesia in patients given interscalene brachial plexus blocks for arthroscopic shoulder surgery. Anesth Analg 2006;103:1278-1282.

18. Mardani-Kivi M, Karimi Mobarakeh M, Keyhani S, Haghighi M, Hashemi-Motlagh K, Saheb-Ekhtiari K. Arthroscopic bankart surgery: Does gabapentin reduce postoperative pain and opioid consumption? A tripleblinded randomized clinical trial. Orthop Traumatol Surg Res 2016;102:549-553.

19. Pham H, Pickell M, Yagnatovsky M, et al. The utility of oral nonsteroidal anti-inflammatory drugs compared with standard opioids following arthroscopic meniscectomy: A prospective observational study. Arthroscopy 2019;35: 864-870.e861.

20. Luo P, Lou J, Yang S. Pain management during rehabilitation after distal radius fracture stabilized with volar locking plate: A prospective cohort study. Biomed Res Int 2018;2018:5786089.

21. Hickernell TR, Lakra A, Berg A, Cooper HJ, Geller JA, Shah RP. Should cannabinoids be added to multimodal pain regimens after total hip and knee arthroplasty? J Arthroplasty 2018;33:3637-3641.

22. Barden J, Edwards J, Moore A, McQuay H. Single dose oral paracetamol (acetaminophen) for postoperative pain. Cochrane Database Syst Rev 2004. CD004602.

23. American Society of Anesthesiologists Task Force on Acute Pain Management. Practice guidelines for acute pain management in the perioperative setting: An updated report by the American Society of Anesthesiologists Task Force on Acute Pain Management. Anesthesiology 2012;1 16:248-273.

24. Chou R, Gordon DB, de Leon-Casasola OA, et al. Management of postoperative pain: A clinical practice guideline from the American Pain Society, the American Society of Regional Anesthesia and Pain Medicine, and the American Society of Anesthesiologists' Committee on Regional Anesthesia, Executive Committee, and Administrative Council. J Pain 2016;17:131-157.

25. Gordon DB, Dahl JL, Miaskowski C, et al. American Pain Society recommendations for improving the quality of acute and cancer pain management: American Pain Society Quality of Care Task Force. Arch Intern Med 2005;165:1574-1580. 
26. Wu Y, Lu X, Ma Y, et al. Perioperative multiple low-dose dexamethasones improves postoperative clinical outcomes after total knee arthroplasty. BMC Musculoskelet Disord 2018;19:428.

27. Sahmeddini MA, Khosravi MB, Farbood A. Comparison of perioperative systemic lidocaine or systemic ketamine in acute pain management of patients with opioid use disorder after orthopedic surgery. J Addict Med 2019;13:220-226.

28. Saini S, McDonald EL, Shakked R, et al. Prospective evaluation of utilization patterns and prescribing guidelines of opioid consumption following orthopedic foot and ankle surgery. Foot Ankle Int 2018;39:1257-1265.

29. Kumar K, Gulotta LV, Dines JS, et al. Unused opioid pills after outpatient shoulder surgeries given current perioperative prescribing habits. Am J Sports Med 2017;45:636-641.

30. Center for Behavioral Health Statistics and Quality. Behavioral health trends in the United States: Results from the 2014 National Survey on Drug Use and Health. 2015. Available at: https://www.samhsa.gov/data/sites/ default/files/NSDUH-FRR1-2014/NSDUH-FRR1-2014. htm.

31. Joris J. Efficacy of nonsteroidal antiinflammatory drugs in postoperative pain. Acta Anaesthesiol Belg 1996;47: 115-123.

32. Ziltener JL, Leal S, Fournier PE. Non-steroidal antiinflammatory drugs for athletes: An update. Ann Phys Rehabil Med 2010;53:278-282.

33. Wheatley BM, Nappo KE, Christensen DL, Holman AM, Brooks DI, Potter BK. Effect of NSAIDs on bone healing rates: A meta-analysis. J Am Acad Orthop Surg 2019;27: e330-e336.

34. Dart RC, Surratt HL, Cicero TJ, et al. Trends in opioid analgesic abuse and mortality in the United States. $N$ Engl J Med 2015;372:241-248. 


\section{Appendix. Telephone Questionnaire}

\section{Call \#1 (24 hours postoperatively)}

1. What is your pain now (from 0 to 10)?

2. Have you taken any oxycodone/acetaminophen since being discharged from the hospital? If yes, how many tablets?

3. What other pain medications are you taking? How many tablets have you taken since being discharged from the hospital?

4. Have you had any of the following side effects: nausea, vomiting, constipation, itching, numbness/tingling in the leg, or weakness in the arm?

5. Any other side effects?

\section{Call \#2 (48 hours postoperatively)}

1. What is your pain now (from 0 to 10 )?

2. Have you taken any oxycodone/acetaminophen since being discharged from the hospital? If yes, how many tablets?
3. What other pain medications are you taking? How many tablets have you taken since being discharged from the hospital?

4. Have you had any of the following side effects: nausea, vomiting, constipation, itching, numbness/ tingling in the leg, or weakness in the arm?

5. Any other side effects?

\section{Call \#3 (1 week postoperatively)}

1. What is your pain now (from 0 to 10 )?

2. Have you taken any oxycodone/acetaminophen since being discharged from the hospital? If yes, how many tablets?

3. What other pain medications are you taking? How many tablets have you taken since being discharged from the hospital?

4. Have you had any of the following side effects: nausea, vomiting, constipation, itching, numbness/ tingling in the leg, or weakness in the arm?

5. Any other side effects?

6. How satisfied are you (from 0 to 10 ) with the pain relief you've received? 